Baltic Astronomy, vol. 5, 207-215, 1996.

\title{
PROGRAM MEGA: TRANSFORMATION OF INSTRUMENTAL MAGNITUDES AND COLOR INDICES TO THE UBVR SYSTEM
}

\author{
V. Andruk and N. Kharchenko
}

Main Astronomical Observatory, Golosiiv, 252650 Kiev, Ukraine

Received August 17, 1995.

\begin{abstract}
The first goal of the photometric part of the MEGA program is the establishment of the sequences of standard magnitudes and color indices by a photoelectric method. From 1984 to 1991, more than 7500 photoelectric UBVR observations of 1141 stars of 4-15.5 magnitudes were carried out at three observatories. The method of reduction of the observations is described. It takes into account color equations between the instrumental and standard magnitudes and colors, the atmospheric extinction and changes of the photometer sensitivity. The accuracy of the photometric data in the final catalog is estimated.
\end{abstract}

Key words: methods: observational - techniques: photometric (UBVR system) - atmospheric effects

\section{INTRODUCTION}

The observational basis of the program MEGA for studying the main meridional section of the Galaxy (Einasto et al. 1985) is the catalogs of astrometric and astrophysical characteristics of stars in more than 60 selected areas (Kharchenko 1983, Schilbach 1988).

In each field we need stellar magnitude standards for the calculation of photographic stellar magnitudes. In the literature we found only a very small number of standard stars in these fields (Andruk \& Kharchenko 1987). Consequently, the first goal of the photometric part of the MEGA program is the creation of standard sequences of magnitudes and color indices by a photoelectric method in all program fields. 


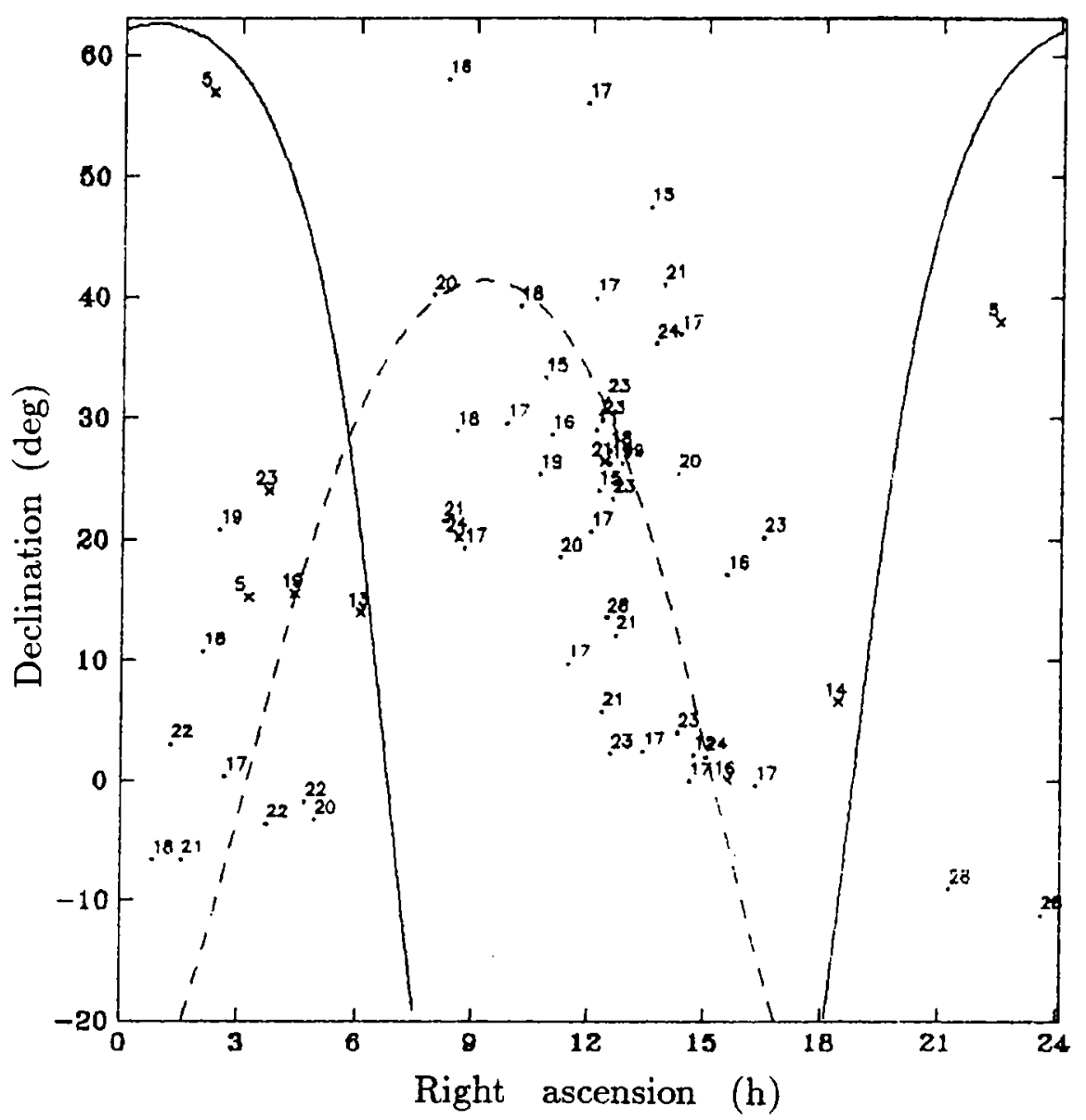

Fig. 1. Distribution of the MEGA areas over the sky in the equatorial coordinate system. Crosses mark those MEGA areas in which we have measured the standard stars and dots mark the remaining MEGA areas. The solid and dashed curves represent the galactic equator and the main meridian, respectively. The numerals indicate the number of standard stars in a given area.

The areas of the MEGA program are distributed over a large part of the sky (Fig. 1). Since we need to set up a uniform photometric system in all the areas, special methods of observations and data reduction should be applied.

From 1984 to 1991, more than 7600 UBVR photoelectric observations of 1141 stars were carried out within the MEGA program. 


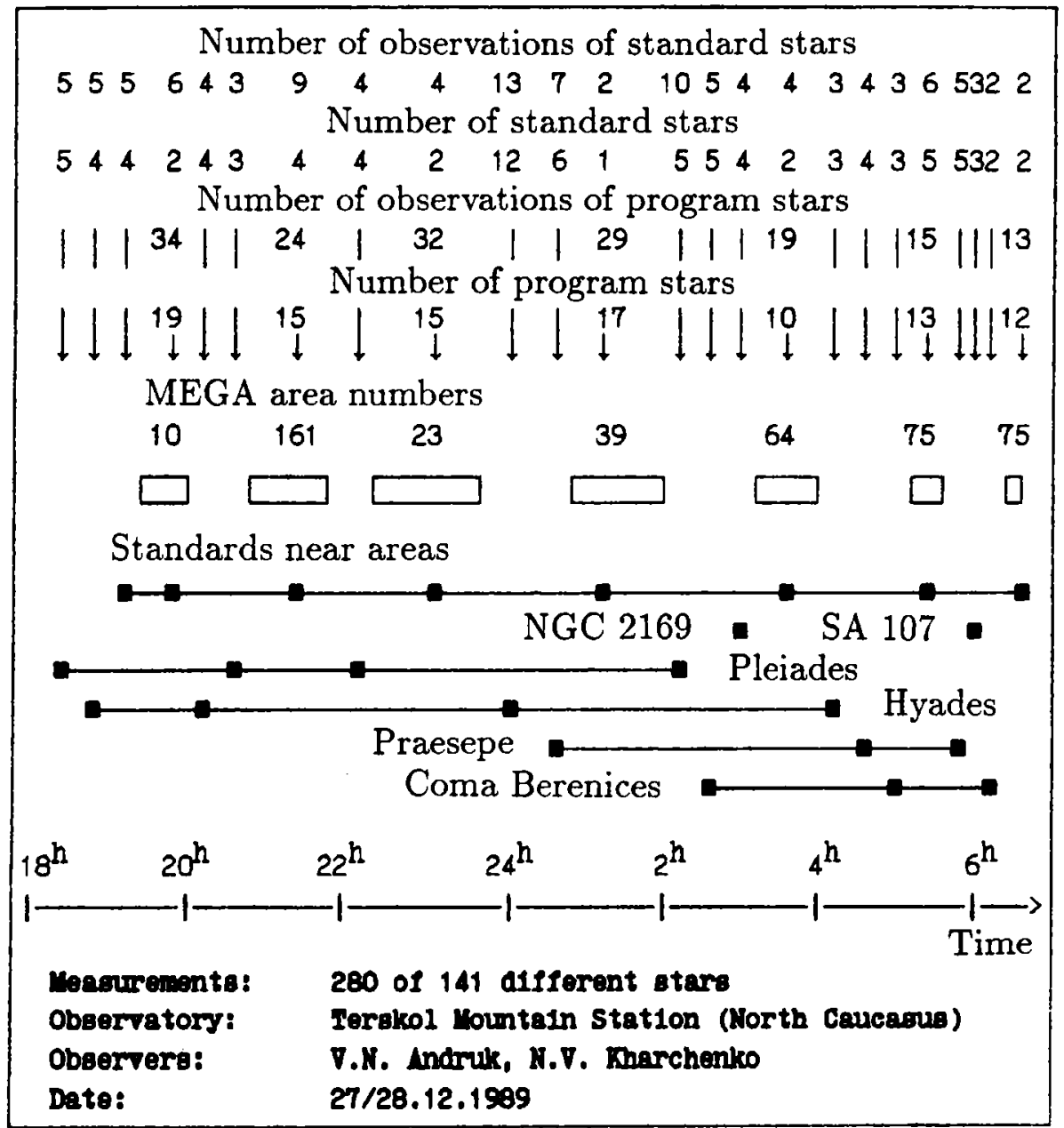

Fig. 2. Time distribution of observations during the longest night, $27 / 28$ December, 1989. The rectangles and filled squares correspond to the observation time of program and standard stars, respectively.

The majority of these observations were made from 1987 to 1991 at the Terskol Mountain Station of the Ukrainian Academy of Sciences located in the North Caucasus. In part, the program stars were observed at the observatories of the Kiev University in Lesniki near Kiev and of the Kazan University in Zelenchuk (North Caucasus).

Standard stars were selected in Kapteyn areas SA 71 and SA 107, the Pleiades, Hyades, Praesepe, Coma Berenices, NGC 6633, 
NGC 2169, $\chi$ Per open clusters, association Lac OB1 and near some of the MEGA fields. The magnitudes and colors of the standard stars were taken from the catalogs of Mendoza (1967), Blanco et al. (1968), Johnson et al. (1966) and Kazanasmas et al. (1981). The standards include 312 stars with known $V$ and $B-V$ data, more than 200 stars with $U-B$ colors and only about 90 stars with $V-R$ colors.

The observations were performed during 105 nights, using three different pulse-counting single-channel photometers (Pugach \& Kovalchuk 1983, Neizvestnyj \& Pimonov 1978, Kleshchenok 1993). On the average, we made 19 observations per standard star and 4 observations per program star. Each program star was measured usually on three different nights. The standard stars were observed at the intervals of about one hour at different air masses $X$ (from 1.0 to 5.5) with hour angles $T$ between $-5 \mathrm{~h}$ and $+6 \mathrm{~h}$. The program stars were observed within $T= \pm 3 \mathrm{~h}$. The amount of the observing time used for standards varied between 20 and 50 per cent on different nights. More than 200 measurements of stars were made on each of the four best nights. During 24 nights, we have made 100 to 200 observations per night. The minimum number of observations per night was 20 . Fig. 2 shows the time distribution of observations during the longest night, 27/28 December, 1989.

\section{REDUCTION OF OBSERVATIONAL DATA}

Observations on each night were considered as an independent set. Their reduction was carried out in three approximations.

(1) In the first approximation, observations were reduced by the following equations :

$$
\begin{aligned}
V_{i} & =a_{1}+b_{1}(b-v)_{i}+k_{1} X_{i}+k_{1}^{\prime}(b-v)_{i} X_{i}, \\
(U-B)_{i} & =a_{2}+b_{2}(u-b)_{i}+k_{2} X_{i}+k_{2}^{\prime}(u-b)_{i} X_{i}, \\
(B-V)_{i} & =a_{3}+b_{3}(b-v)_{i}+k_{3} X_{i}+k_{3}^{\prime}(b-v)_{i} X_{i}, \\
(V-R)_{i} & =a_{4}+b_{4}(v-r)_{i}+k_{4} X_{i}+k_{4}^{\prime}(v-r)_{i} X_{i},
\end{aligned}
$$

where $X$ is the air mass, $i=1,2, \ldots, n$ and $n$ is the number of all measurements of standards per night, $m$ is the number of standards. For the i-th standard star, the values of $\left.\left.V_{i},(U-B)_{i}\right),(B-V)_{i}\right)$ and $\left(V-R_{i}\right)$ are known, the coefficients $a_{j}, b_{j}, k_{j}$ and $k_{j}^{\prime}(j=1,2,3,4)$ are the unknowns which are being determined separately for each night. These coefficients characterize the connection between the 
instrumental $v, u-b, b-v, v-r$ and the standard $V, U-B, B-V, V-R$ systems, the atmospheric extinction $\left(k_{j}\right)$ and the dependence of the extinction on the observed color index of the star $\left(k_{j}^{\prime}\right)$. For some nights, the determination of the coefficients $k_{j}^{\prime}$ was not possible due to the insufficient number of the standard stars observed. In these cases, the system of equations (1) was solved without the coefficients $k_{j}^{\prime}$. The least squares solution of the system of equations (1) was carried out by excluding the stars exceeding $3 \sigma$ deviation. The newly determined individual stellar magnitudes and colors of standard and program stars were averaged over all observations and all nights for a given star so that we got $\overline{V_{k}}, \overline{(U-B)_{k}}, \overline{(B-V)_{k}}$ and $\overline{(V-R)_{k}}$.

(2) In the second approximation, the obtained results, $\overline{V_{k}}$, $\overline{(U-B)_{k}}, \overline{(B-V)_{k}}$ and $\overline{(V-R)_{k}}$, for all stars with $V<13$ mag were used as an extended photometric system of standards (secondary standard stars). The solution of system (1) was repeated separately for each night then using a larger number of standard stars. After that we obtained again the mean magnitude and colors of each star by averaging all $V_{i},(U-B)_{i},(B-V)_{i},(V-R)_{i}$ belonging to that star.

(3) In the third approximation, we took into account the changes of the mean atmospheric extinction and of the sensitivity of the photometer with time $\tau$, the energy distribution in stellar spectra (described by the coefficients $\beta$ in the relation of color indices; for more details see Pugach \& Kovalchuk 1983) and the changes of the air mass $X$ due to tidal effects in the Earth's atmosphere due to the Moon and the Sun (Andruk et al. 1991). The final equations for each observation have the form:

$$
\begin{aligned}
V_{i}= & a_{1}+b_{1} v_{i}+k_{1} X_{i}+k_{1}^{\prime}(b-v)_{i} X_{i}+\alpha_{1}^{\prime} \cos \left(\tau_{i}-\bar{\tau}\right)+ \\
& \alpha_{1}^{\prime \prime} \ln \tau_{i}+\beta_{1}(b-v)_{i} /(v-r)_{i}+\gamma_{1}^{\prime} \cos L_{i}+\gamma_{1}^{\prime \prime} \cos S_{i}, \\
(U-B)_{i}= & a_{2}+b_{2}(u-b)_{i}+k_{2} X_{i}+k_{2}^{\prime}(u-b)_{i} X_{i}+\alpha_{2}^{\prime} \cos \left(\tau_{i}-\bar{\tau}\right)+ \\
& \alpha_{2}^{\prime \prime} \ln \tau_{i}+\beta_{2}(u-b)_{i} /(v-r)_{i}+\gamma_{2}^{\prime} \cos L_{i}+\gamma_{2}^{\prime \prime} \cos S_{i}, \\
(B-V)_{i}= & a_{3}+b_{3}(b-v)_{i}+k_{3} X_{i}+k_{3}^{\prime}(b-v)_{i} X_{i}+\alpha_{3}^{\prime} \cos \left(\tau_{i}-\bar{\tau}\right)+ \\
& \alpha_{3}^{\prime \prime} \ln \tau_{i}+\beta_{3}(b-v)_{i} /(v-r)_{i}+\gamma_{3}^{\prime} \cos L_{i}+\gamma_{3}^{\prime \prime} \cos S_{i}, \\
(V-R)_{i}= & a_{4}+b_{4}(v-r)_{i}+k_{4} X_{i}+k_{4}^{\prime}(v-r)_{i} X_{i}+\alpha_{4}^{\prime} \cos \left(\tau_{i}-\bar{\tau}\right)+ \\
& \alpha_{4}^{\prime \prime} \ln \tau_{i}+\beta_{4}(b-v)_{i} /(v-r)_{i}+\gamma_{4}^{\prime} \cos L_{i}+\gamma_{4}^{\prime \prime} \cos S_{i}, \quad(2)
\end{aligned}
$$

where $\tau_{i}$ is the mean time of the $i$-th observation, $\bar{\tau}=\left(\tau_{\text {end }}-\tau_{\text {start }}\right) / 2$ where $\tau_{\text {start }}$ is the time of the beginning and $\tau_{\text {end }}$ is the time of the end of observations for each night, $L$ and $S$ are the angular distances on the sky between the observed star and the tidal maxima of the 
Moon and of the Sun, respectively. Here we have $i=1,2, \ldots, n$, where $n$ is the general number of observations of secondary standard stars used in the third reduction step.

The form of Eqs. (2) was chosen empirically from the analysis of the residuals $\Delta U_{i}=U_{i}-\overline{U_{k}}$ and similar residuals $\Delta B_{i}, \Delta V_{i}$ and $\Delta R_{i}$ according to the results of the reduction for photometric nights of the best quality (about 30). Here, $\overline{U_{k}}, \overline{B_{k}}, \overline{V_{k}}, \overline{R_{k}}$ are the mean magnitudes determined for the $k$-th star and $U_{i}, B_{i}, V_{i}, R_{i}$ are the magnitudes obtained from a single measurement of the $k$-th star. The terms $\cos \left(\tau_{i}-\bar{\tau}\right)$ (with radians as an argument) and $\ln \tau_{i}$ (with time in hours) were found empirically to give the best fit to the data. The determined magnitudes and colors were finally averaged again over all measurements of all nights.

In all three reduction steps, the averaging of magnitudes and colors of a given star was carried out applying the criterion of $3 \sigma$ deviation from the mean. For a better exclusion of outliers, instead of the arithmetic mean, we computed the weighted mean, the weights being the deviations of individual values from the arithmetic mean:

$$
\bar{m}=\frac{\sum_{i=1}^{N} \prod_{\substack{l=1 \\ l \neq i}}^{N} m_{i} d_{l}^{2}}{\sum_{i=1}^{N} \prod_{\substack{l=1 \\ l \neq i}}^{N} d_{l}^{2}},
$$

where $m_{i}$ is a single value obtained from one measurement of a given star, $N$ is the general number of values determined over all nights for the star and $d_{l}=m_{l}-m$ is a difference between a single measurement and the arithmetic mean $m$. Eq. (3) was iteratively applied three times.

\section{ACCURACY OF THE CATALOG AND CONCLUSIONS}

The rms errors of the magnitude and colors for each star were determined as

$$
\sigma_{k}=\sqrt{\frac{\sum_{i=1}^{N}\left(m_{i}-\bar{m}\right)^{2}}{N(N-1)}},
$$

where $N$ is the number of observations for the $\mathrm{k}$-th star. These errors are given in the catalog of observations (Andruk et al. 1995).

In comparison to the first reduction step, the rms errors of the final solution were improved by about 20 to 40 per cent. Fig. 3 shows the mean internal rms errors of $V, U-B, B-V$ and $V-R$ data 
obtained after all three reduction steps in dependence on different magnitude intervals.

External rms errors were obtained from the comparison of three catalogs: our catalog, $\mathrm{C} 1$, the catalog of $W B V R$ magnitudes of Kornilov et al. (1991), C2, and the data for the primary standards, C3. Since the ultraviolet magnitude $W$. of Kornilov et al. has a different response function in comparison to Johnson's ultraviolet magnitude $U$, a comparison of the color indices from the $\mathrm{C} 2$ cata$\log ,(W-B)_{2}$, with those in the other catalogs, $(U-B)_{1}$ and $(U-B)_{3}$, was not made. Since all three catalogs are in the same photometric system, they can be considered as independent catalogs only from the point of view of different methods of taking into account the atmospheric extinction and of different reduction equations from the instrumental to the standard system.

Table 1 gives the external rms errors $\varepsilon$ of the three catalogs after the removal of systematic differences.

Table 1. External random errors of the catalogs C1, C2 and C3

\begin{tabular}{cccc}
\hline & $\varepsilon_{C 1}$ & $\varepsilon_{C 2}$ & $\varepsilon_{C 3}$ \\
\hline$V$ & \pm 0.014 & \pm 0.009 & \pm 0.019 \\
$U-B$ & \pm 0.026 & & \pm 0.026 \\
$B-V$ & \pm 0.012 & \pm 0.007 & \pm 0.011 \\
$V-R$ & \pm 0.016 & \pm 0.015 & \pm 0.010 \\
\hline
\end{tabular}

Our catalog includes 1141 stars and provides photometric sequences in 47 selected areas of the MEGA program, four areas near the North Galactic Pole and some open clusters in the magnitude $V$ interval from 4.5 to $15.5 \mathrm{mag}$. The catalog contains equatorial coordinates for equinox of 1950.0 (Kharchenko 1989) with an accuracy which is sufficient for identification.

\section{REFERENCES}

Andruk V. N., Kharchenko N. V. 1987, Kinematics and Physics of Celestial Bodies, Kiev, 3, 61

Andruk V.N., Kharchenko N. V., Schilbach E., Scholz R.-D. 1995, Astron.

Nachrichten, 316, 225 

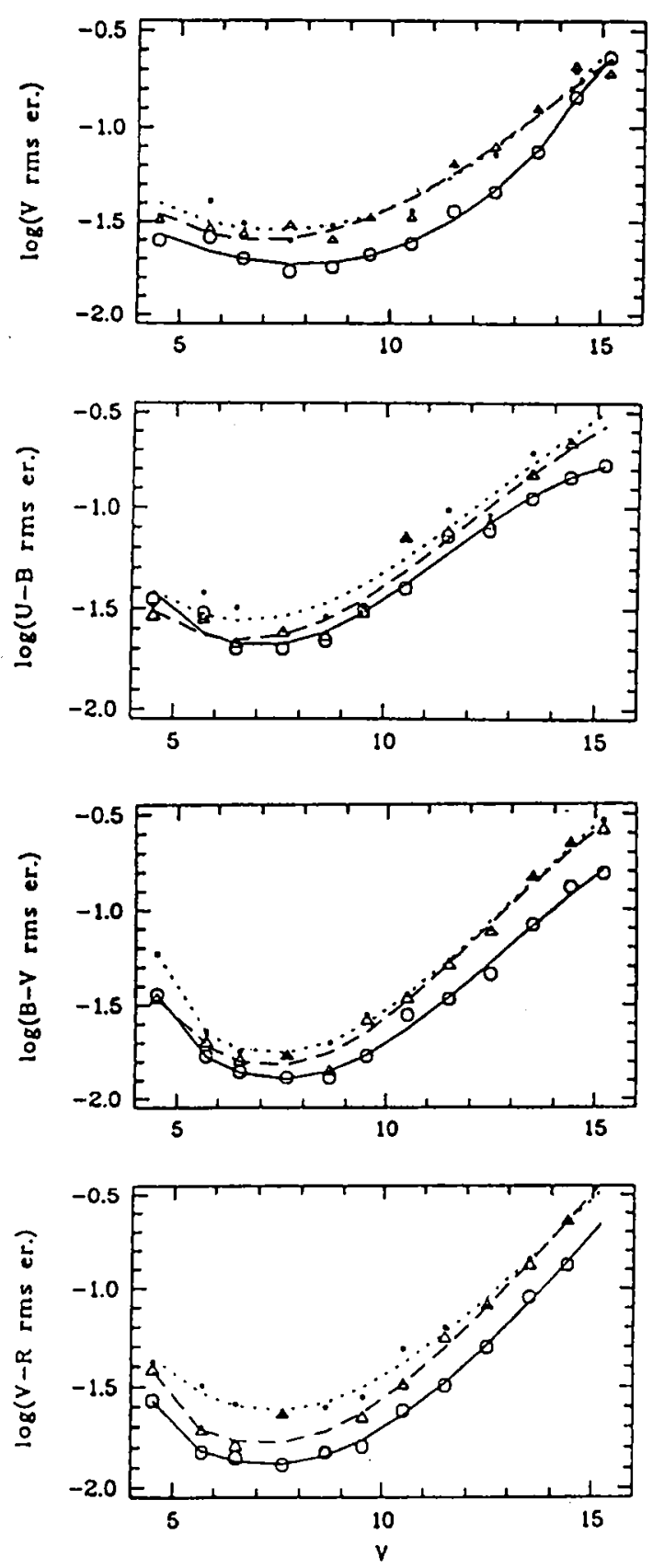

Fig. 3. Mean internal rms errors of $V, U-B, B-V$ and $V-R$ as a function of magnitude. Dots and dotted lines are for the first approximation, triangles and dashed lines are for the second approximation and open circles and solid lines are for the third approximation of the reduction. 
Andruk V.N., Kitkin V.N., Kleshshenok V.V., Kharchenko N. V. 1991, in Problems of Astrometry and Space Geodynamics (Proc. Internat. Workshop), ed. N. V. Kharchenko, Naukova Dumka Publ. House, Kiev, p. 28

Blanco V.M., Demers S., Douglas G. G., Fitzgerald M. P. 1968, Publ. US Naval Obs., 2nd series, vol. 21

Einasto J., Malyuto V.D., Kharchenko N.V. 1985, Astron. Circular, Moscow, No. 1384, 1

Johnson H.L., Mitchell R.I., Iriarte B., Wisniewski W.Z. 1966, Comm. Lunar and Plan. Lab., Tucson, Arizona, 4, No. 63, 99

Kazanasmas M.S., Zavershneva M. A., Tomak L.F. 1981, Atlas and Catalogue of Stellar Magnitudes of Photoelectric Standards, Naukova Dumka Publishers, Kiev

Kharchenko N. V. 1983, Astrometry and Astrophysics, No. 49, 61

Kharchenko N. V. 1989, Kinematics and Physics of Celestial Bodies, 5, 9

Kleshchenok V.V. 1993, Photoelectric Photometer of the Kiev Observatory, deposited paper No. 481-B93, Moscow, United Institute of Scientific and Technical Information

Kornilov V. G., Volkov I. M., Zakharov A. I., Kozyreva V.S., Kornilova L. N., Krutyakov A.N., Krylov A.V., Kusakin A.V., Leontiev S.E., Mironov A. V., Moshkaliov V. G., Pogrosheva T. M., Sementsov V.N., Khaliullin Kh.F. 1991, Catalogue of WBVR magnitudes of Bright Stars in the Northern Sky, Trudy Astron. Inst. Sternberg, Moscow University, vol. 63

Mendoza E. E. 1967, Bol. Obs. Tonantzintla y Tacubaya, 4, 149

Neizvestnyj S.I., Pimonov A.A. 1978, Comm. Special Astrophys. Obs., No. 23, 56

Pugach A.F., Kovalchuk G. U. 1983, Variable Stars, Moscow, 22, 9 Schilbach E. 1988, in Mapping the Sky (IAU Symp. No. 133), eds. S. Debarbat et al., Kluwer Academic Publishers, Dordrecht, p. 451 
